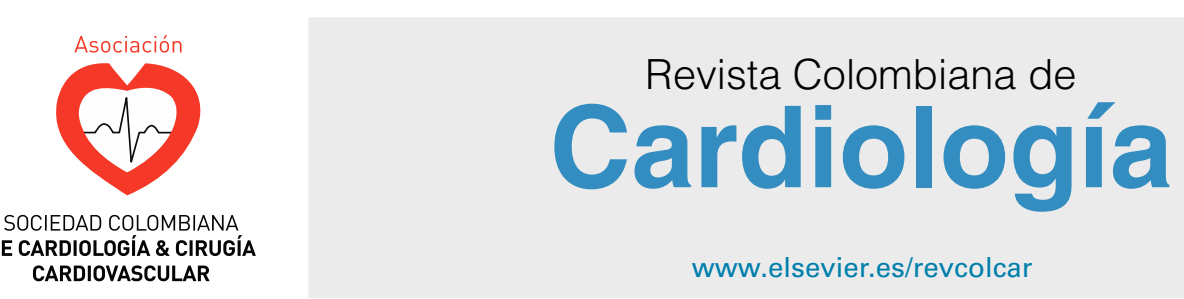

CARDIOLOGÍA DEL ADULTO - PRESENTACIÓN DE CASOS

\title{
Identificación de variante anatómica en la ablación exitosa de aleteo auricular típico luego de cirugía valvular: utilidad de la ecografía intracardiaca
}

\author{
Jhan Altamar*, Carlos Tapias, Luis Sáenz, Diego Rodríguez y Juan Montenegro
}

Centro internacional de arritmias, Fundación Cardioinfantil, Universidad de la Sabana, Bogotá, Colombia

Recibido el 17 de enero de 2017; aceptado el 11 de agosto de 2018

Disponible en Internet el 21 de diciembre de 2018

\section{PALABRAS CLAVE}

Catéter ;Ablación;

Aleteo auricular;

Válvula tricúspide

\section{KEYWORDS}

Catheter;

Ablation;

Atrial flutter;

Tricuspid valve
Resumen Se hizo ablación tridimensional de aleteo auricular, dependiente del istmo cavotricuspídeo, en un paciente con cirugía previa de válvula tricúspide. El uso de ecocardiografía intracardiaca permitió identificar una estructura sacular en el aspecto septal del istmo. Después de la ablación en este punto, el aleteo terminó y se confirmó bloqueo bidireccional. Este reporte sugiere, además, que el miocardio auricular ligado al anillo valvular, sirve como vía de conducción crítica del aleteo auricular.

(c) 2018 Sociedad Colombiana de Cardiología y Cirugía Cardiovascular. Publicado por Elsevier España, S.L.U. Este es un artículo Open Access bajo la licencia CC BY-NC-ND (http:// creativecommons.org/licenses/by-nc-nd/4.0/).

Identification of an anatomical variant in the successful ablation of a typical atrial flutter after valve surgery: Usefulness of intracardiac echocardiography

Abstract A three-dimensional ablation of an atrial flutter, dependent on the cavo-tricuspid isthmus, was performed on a patient with previous tricuspid valve surgery. The use of intracardiac echocardiography enabled a saccular structure to be identified in the septal aspect of the isthmus. After the ablation in this point, the flutter stopped and a two-directional block was confirmed. This report suggests, furthermore, that the atrial myocardium together with the valvular ring, serves as a critical conduction pathway of the atrial flutter.

(c) 2018 Sociedad Colombiana de Cardiología y Cirugía Cardiovascular. Published by Elsevier España, S.L.U. This is an open access article under the CC BY-NC-ND license (http:// creativecommons.org/licenses/by-nc-nd/4.0/).

\footnotetext{
* Autor para correspondencia.

Correo electrónico: jcarlomed@gmail.com (J. Altamar).
} 


\section{Introducción}

El aleteo auricular típico es una arritmia común, en la que la ablación con catéter se convierte en una estrategia de tratamiento segura y con alta tasa de éxito. Sin embargo, este abordaje ha sido poco reportado en pacientes con cirugía de válvula tricúspide, posiblemente por el incremento potencial de los riesgos durante la ablación cerca de la prótesis ${ }^{1}$. En estos casos, el uso de ecografía intracardiaca, permite realizar el procedimiento de manera segura y además ofrece información que no es posible obtener con otros métodos.

\section{Caso}

Paciente de sexo masculino, de 31 años de edad, con antecedente de enfermedad renal crónica estadio $V$ en terapia de reemplazo renal, quien tres meses después de ser realizadas plastia de válvula mitral y tricúspide con anillo, desarrolló aleteo auricular típico antihorario (fig. 1). En el laboratorio de Electrofisiología bajo anestesia local y abordaje venoso bifemoral se avanzaron introductores cortos y camisa deflectable AGILIS ${ }^{T M}$ de St. Jude medical. Se posicionó sonda de ecografía intracardiaca de 10F ACUSON AcuNav $^{\top M}$ de Siemens, en la aurícula derecha (home view), catéter decapolar, el cual se ubicó en el seno coronario y catéter de ablación Biosense Webster Thermocool SF. Se confirmó mediante la realización de encarrilamiento desde el aspecto septal y lateral del istmo cavotricuspídeo, que el aleteo era dependiente del mismo. Usando sistema de mapeo tridimensional Carto 3 , se realizó reconstrucción electro-anatómica de la aurícula derecha punto a punto con el catéter de ablación y mapa de activación durante aleteo auricular, teniendo como electrograma de referencia el seno coronario (Fig. 2), pudiéndose observar el punto de activación temprana (early meets late) en el istmo cavotricuspídeo. La eliminación del aleteo no podía hacerse después de $120 \mathrm{~min}$ de procedimiento, a pesar de repetidas aplicaciones de radiofrecuencia a lo largo de istmo (utilizando hasta $40 \mathrm{~W}$ de poder, 40 grados centígrados de temperatura e irrigación 15 a $37 \mathrm{ml} / \mathrm{min}$ ). Mediante la sonda de ecografía intracardiaca se pudo visualizar una depresión en el aspecto septal del istmo (fig. 3B), el cual es un defecto residual asociado a la plastia tricúspide. Al inyectar medio de contraste sobre el istmo cavo tricúspide a través del catéter de ablación el cual posee microporos de irrigación en su punta y consiguiendo estabilización de este por medio de la camisa deflectable, se pudo visualizar el receso (fig. 3A). El aleteo pudo ser terminado con aplicación de radiofrecuencia dentro del receso (fig. 2). Se confirmó la ubicación y la relación espacial del defecto mediante ecocardiografía transesofágica tridimensional en el postoperatorio (fig. 2D).

\section{Discusión}

El aleteo auricular típico es una arritmia auricular en la que el mecanismo electrofisiológico es una combinación de conducción lenta a nivel del istmo cavotricuspídeo y bloqueo de conducción anatómico o funcional a lo largo de la crista terminalis y la válvula de Eustaquio. Teniendo en cuenta el sustrato anatómico bien definido y la resistencia farmacológica, la ablación con catéter emergió en la década pasada como una estrategia de tratamiento de primera línea, segura y efectiva. Sin embargo, pueden presentarse dificultades para la ablación, las cuales están relacionadas con presencia de músculos pectíneos, válvulas de Eustaquio prominentes y bolsas o recesos $^{2}$; estos últimos son el mayor obstáculo para crear línea de lesión de radiofrecuencia después de cirugía de válvula tricúspide ${ }^{3}$. En estos defectos anatómicos pueden anclarse fibras musculares que sirvan como vía crítica del circuito o simplemente
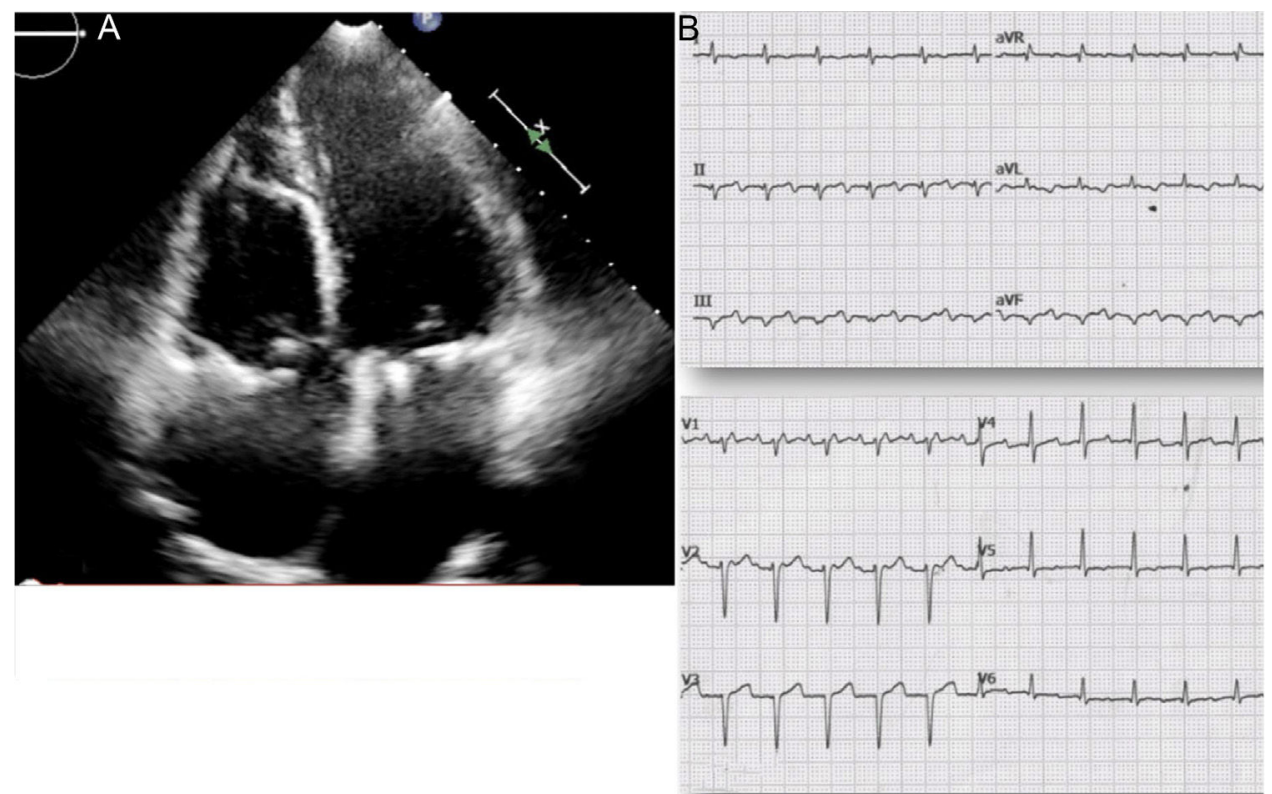

Figura 1 A. Ecocardiograma trastorácico, proyección 4 cámaras. Se pueden observar zonas de hiperrefringencia que corresponden a los anillos mitral y tricúspide por plastias valvulares. B. Electrocardiograma de 12 derivadas, aleteo auricular común con conducción 2:1. 


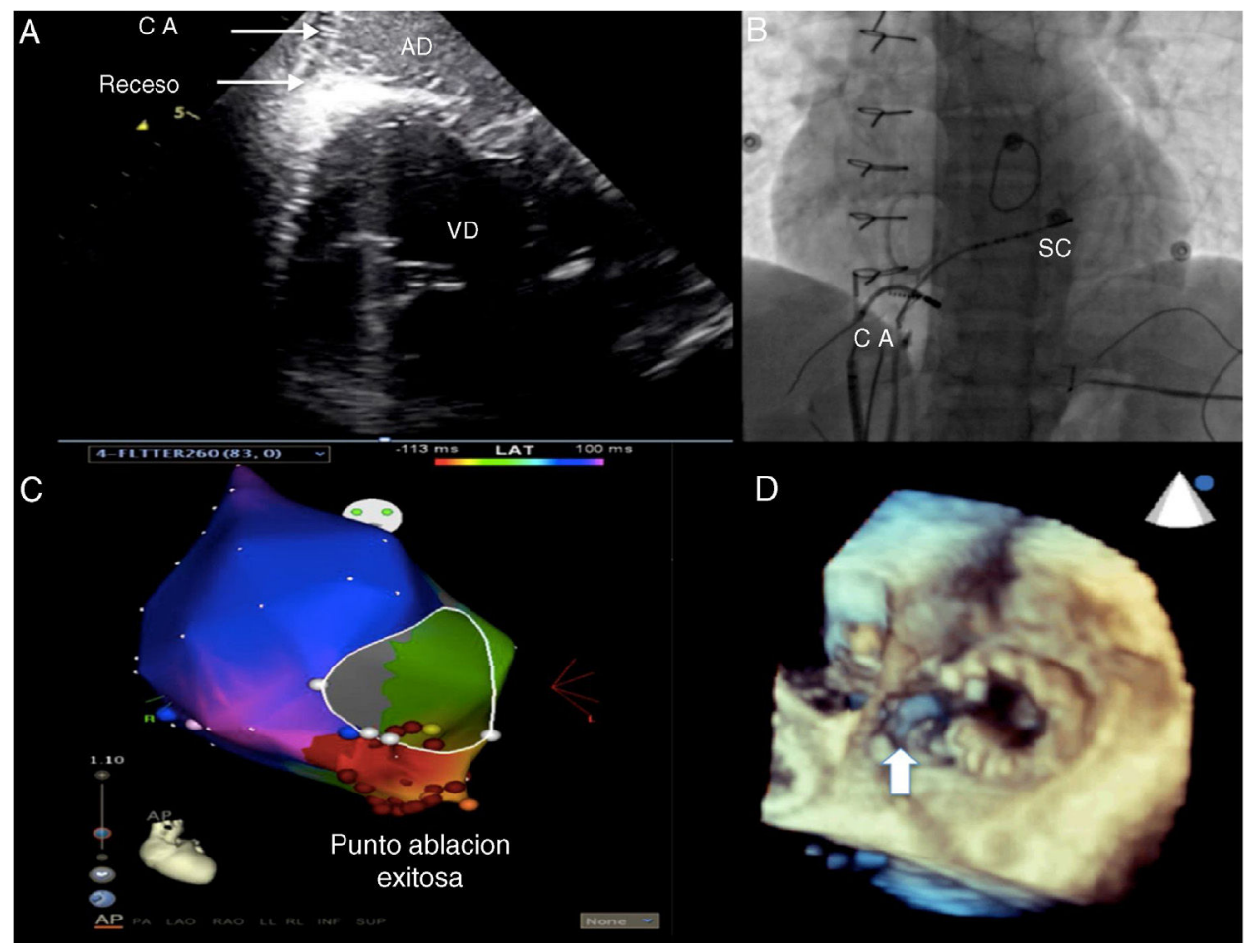

Figura 2 A. Imagen de eco intracardíaco durante ablación. Se observa la punta del catéter de ablación en el receso del istmo cavotricúspide. AD: aurícula derecha, VD: ventrículo derecho. B. Fluoroscopia cardíaca en proyección oblicua anterior izquierda durante la ablación. El catéter de ablación se encuentra posicionado a las 5 horas del reloj con la punta en el receso, el cual fue el punto de ablación exitoso. C. Mapa activación donde se observa la zona de activación temprana (área de color roja) y la secuencia de activación de la aurícula derecha desde lo más precoz (zona de color rojo) hasta la zona de activación tardía (zona de color morado) en sentido anti horario. Además, se observa el punto de ablación exitosa, marcado con un punto naranja, el cual corresponde al receso en el istmo cavo tricúspide. CA: catéter de ablación, SC: seno coronario. D. Ecocardiograma transesofágico con reconstrucción 3D donde se señala con la flecha blanca el receso a nivel septal en el istmo cavotricúspide.

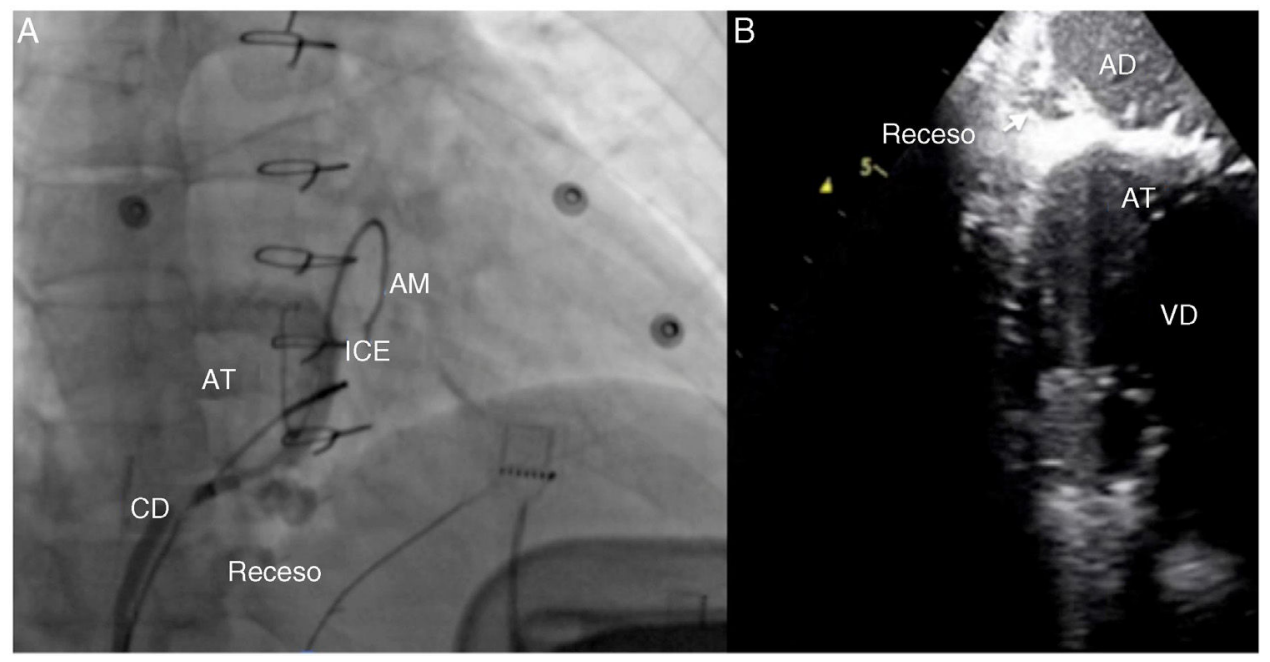

Figura 3 A. Imagen fluoroscópica cardíaca en proyección oblicua anterior derecha; Se pueden observar los anillos valvulares y el receso o saco en el istmo cavotricuspídeo al realizar la inyección de medio de contraste a través del catéter de ablación el cual es posicionado y estabilizado con una camisa deflectable. AM: anillo mitral, AT: anillo tricúspide, CD: camisa deflectable, ICE: sonda de eco intracardíaco. B. Imagen de ecografía intracardíaca, en la que se observa receso en el istmo cavotricuspídeo en su aspecto septal. AD: aurícula derecha, AT: anillo tricúspide, VD: ventrículo derecho. 
generar dificultad para completar la línea de bloqueo. La identificación de estos defectos puede ser determinante en el éxito de la ablación. En estos casos la fluoroscopia por sí sola no permite la identificación de marcas anatómicas específicas y es aquí donde la ecocardiografía intracardiaca tiene un papel crucial en el éxito de la ablación.

La guía fluoroscópica en intervenciones complejas tiene limitaciones significativas, pudiendo presentarse complicaciones por una maniobrabilidad subóptima de catéteres, a riesgo de perforación cardíaca, o simplemente tiempos de procedimiento prolongados ${ }^{4}$. La ecografía intracardiaca aprovecha al máximo las habilidades de la ecografía en procedimientos de electrofisiología. Se utiliza exclusivamente como guía en los procedimientos, pues se asocia con bajo riesgo global, describiéndose solo como una potencial causa de arritmias transitorias por efecto mecánico ${ }^{5}$. Las imágenes de ecocardiografía intracardíaca pueden ser recolectadas de múltiples planos y luego superpuestas a mapas electro-anatómicos existentes, como la tomografía computarizada o resonancia magnética existente ${ }^{6}$. Así, la ecografía intracardíaca puede utilizarse de manera concomitante con otras modalidades de imagen para mejorar la representación espacial de la anatomía cardíaca y las posiciones del catéter. Permite ver la calidad del contacto del catéter y de la superficie del tejido antes y durante el suministro de la energía de radiofrecuencia y como en el caso expuesto, defectos o variantes anatómicas relacionadas con cirugía previa. En este caso, en el postoperatorio se hizo ecocardiograma transesofágico tridimensional de manera académica para demostrar de forma más detallada la observación realizada durante el procedimiento. A pesar de que esta herramienta está disponible en nuestro centro y que podría realizarse en tiempo real, la ecografía intracardíaca parece tener algunas ventajas sobre el ecocardiograma transesofágico, como tiempos de intervención y de salas más cortos ${ }^{7}$, con la que además se reduce la exposición a radiación, ofreciendo beneficios directos al paciente y al operador.

\section{Conclusión}

En el caso reportado, la ecografía intracardiaca fue de gran utilidad, pues facilitó la identificación del receso en el istmo cavotricuspídeo y la ablación exitosa del aleteo. En pacientes con cirugía de válvula tricúspide, esta herramienta ofrece información imposible de obtener con otros métodos.

\section{Conflicto de intereses}

Ninguno.

\section{Agradecimientos}

Servicio de Electrofisiología/Centro internacional de arritmias Andrea Natale.

\section{Bibliografía}

1. Nordbeck P, Bauer WR, Ritter O. Ablation of atrial flutter in a patient with a tricuspid valve replacement after endocarditis. Pcing Clin Eletrophysiol. 2009;32:1237-9.

2. Asirvatham SJ. Correlative anatomy and electrophysiology for the interventional electrophysiologist: right atrial flutter. J Cardiovasc Electrophysiol. 2009;20:113-22.

3. Goto E, Nakamura K, Sasaki T, Naito S. Successful ablation of cavotricuspid isthmus-dependent atrial flutter guided by contact force vector in a patient after a tricuspid valve replacement. Indian Pacing Electrophysiol. 2014;14:306-8.

4. Bartel T, Mu S. Device closure of interatrial communications: peri-interventional echocardiographic assessment. Eur Heart J. 2013;14:618-24.

5. Hijazi ZM, Shivkumar K, Sahn DJ. Intracardiac echocardiography during interventional and electrophysiological cardiac catheterization. Circulation. 2009;119:587-96.

6. Burke MC, Roberts MJD, Knight BP. Integration of cardiac imaging and electrophysiology during catheter ablation procedures for atrial fibrillation. J Elctrocardiol. 2006;39 4 suppl:188-92.

7. Alboliras ET, Hijazi ZM. Comparison of costs of intracardiac echocardiography and transesophageal echocardiography in monitoring percutaneous device closure of atrial septal defect in children and adults. Am J Cardiol. 2004;94:690-2. 